\title{
Anabases
}

ANABASES Traditions et réceptions de l'Antiquité

$9 \mid 2009$

Varia

\section{Étude préliminaire sur les réseaux de correspondants européens de Joseph Déchelette}

Sandra Péré-Noguès

\section{(2) OpenEdition}

1 Journals

Édition électronique

URL : http://journals.openedition.org/anabases/440

DOI : 10.4000/anabases.440

ISSN : 2256-9421

Éditeur

E.R.A.S.M.E.

Édition imprimée

Date de publication : 1 mars 2009

Pagination : 205-220

ISSN : 1774-4296

\section{Référence électronique}

Sandra Péré-Noguès, "Étude préliminaire sur les réseaux de correspondants européens de Joseph

Déchelette », Anabases [En ligne], 9 | 2009, mis en ligne le 01 mars 2012, consulté le 20 octobre 2019.

URL : http://journals.openedition.org/anabases/440 ; DOI : 10.4000/anabases.440

(C) Anabases 
Anabases 9 (2009), p. 205-220.

\section{Étude préliminaire sur les réseaux de correspondants européens de Joseph Déchelette ${ }^{1}$}

SANDra PÉrÉ-NoguÈs

Si Joseph DéChelette (1862-1914) est aujourd'hui connu comme l'auteur du remarquable Manuel d'archéologie préhistorique, celtique et gallo-romaine ${ }^{2}$, il fut aussi, en son temps, un grand savant dont la réputation dépassa largement les frontières du territoire français. Sa correspondance passive, avec sa bibliothèque, constitue une part essentielle du patrimoine du Musée de la ville de Roanne, mais en l'absence d'une valorisation $\mathrm{du}$ fonds documentaire, la correspondance n'a pas encore fait l'objet d'une recherche historiographique systématique ${ }^{3}$. Depuis presque deux ans, nous avons engagé un

1 Ce programme de recherche, soutenu par la bibliothèque de Roanne, le laboratoire TRACES et par le Centre Européen d'Archéologie de Bibracte devrait aboutir à une valorisation complète de la correspondance passive et, à plus ou moins long terme, à la récupération de la correspondance active de l'archéologue roannais. Nos remerciements les plus chaleureux vont à Pierre-Yves Milcent et à Micheline Petiot, bibliothécaire de Roanne, sans qui ce projet n'aurait pu voir le jour. Enfin, toute notre gratitude va à Corinne Bonnet pour sa lecture et ses précieux conseils.

2 Joseph DÉCHELETTE, Manuel d'archéologie préhistorique, celtique et gallo-romaine, Paris, Picard, 1908-1914 (4 volumes).

3 Parmi les études sur Joseph Déchelette, il faut citer l'intéressante thèse de Marie-Suzanne Binétruy, De l'art roman à la Préhistoire. Des sociétés savantes à l'Institut, itinéraires de Joseph Déchelette, Lyon, 1994. Voir aussi François DéCheleTte, Livre d'or de Joseph Déchelette (Centenaire 1862-1962), Roanne, 1962. Cet ouvrage fut réalisé à la demande de Mme Déchelette par un des cousins de la famille pour commémorer le centenaire de la naissance de l'archéologue roannais. Il rassemble des témoignages contemporains de son époque (extraits d'ouvrages, comptes rendus ou lettres) et des textes de savants français et étrangers qui avaient été sollicités pour cette commémoration. 
programme de recherches sur le corpus épistolaire en nous limitant, pour l'instant, à la correspondance venant des pays étrangers. Cette première étape devrait donner lieu à d'autres études plus spécifiques sur les " réseaux " scientifiques européens et nationaux qui ont promu la Protohistoire au tournant des $\mathrm{XIX}^{\mathrm{e}}$ et $\mathrm{XX}^{\mathrm{e}}$ siècles.

La correspondance conservée à la bibliothèque du Musée de Roanne rassemble une grande partie des lettres envoyées à Joseph Déchelette, soit près de trois mille lettres qui furent soigneusement reliées en quarante-trois volumes in-quarto à l'initiative de Madame Déchelette ${ }^{4}$. Une autre partie est composée de la correspondance active (quelques centaines de lettres) mais elle n'a pas été recensée de manière précise.

Le but de la présente enquête est de donner quelques repères sur les différents réseaux et correspondants avec lesquels Joseph Déchelette était en relation. L'étude de la correspondance passive permet, en effet, une analyse approfondie du " réseau personnel » de Joseph Déchelette, cette notion de " réseau personnel » étant définie par les sociologues comme "l'ensemble formé d'un individu, des individus qui sont en relation directe avec lui et des relations que ces individus entretiennent les uns avec les autres ${ }^{5}$ ". C'est en définitive à la découverte d'un milieu de savants et d'érudits que cette analyse invite, un milieu avec ses règles de fonctionnement et de sociabilité, ses stratégies et ses " écoles » de pensée. Cette enquête devrait donc contribuer à apprécier la position qu'assuma Joseph Déchelette dans ce " petit monde " dont il fut l'un des plus illustres représentants.

Mais pour saisir ce que l'œuvre et son auteur ont pu représenter pour la communauté scientifique de l'époque, nous commencerons « à rebours » en choisissant délibérément d'évoquer les divers hommages qui furent rendus suite à la disparition brutale - mais ô combien honorable - du conservateur de Roanne ${ }^{6}$.

$4 \quad$ Le $43^{\mathrm{e}}$ volume regroupe notamment toutes les lettres de condoléances. Un petit nombre de lettres qui furent trouvées après la constitution des tomes est encore dispersé dans plusieurs dossiers et devrait être prochainement archivé.

5 Voir à ce sujet P. Mercklé, Sociologie des réseaux sociaux, Paris, 2008, p. 35. Il s’inspire directement de la définition formulée dans les années 30 par J.L. Moreno à propos de "l'atome " social. Pour l'approche analytique, notamment par une analyse égocentrée: voir C. Mercier et C. ZalC, Méthodes quantitatives pour l'historien, Paris, 2008, p. 87.

6 Rappelons que Joseph Déchelette, intégré à sa demande dans un régiment d'active, et ce malgré ses 52 ans, est mort de ses blessures après une attaque lancée à la tête de sa compagnie, le 4 octobre 1914, près de Vingré dans l'Aisne. C. Jullian à $\mathrm{M}^{\text {me }}$ Déchelette, lettre du 25 octobre 1914: "Il a été notre maître en tout, en science, en vertu, en courage. Soyez fière de son souvenir, soyez glorieuse de son nom. " Dans l'hommage qu'il rédige pour la Revue des Études Anciennes en 1914 (p. 417), il écrit comme en écho: "Et ce que le vaillant disparu a fait, dans sa vie, pour la science de notre pays, dans sa mort, pour son salut, doit lui valoir un renom immortel. » 


\section{La reconnaissance officielle d'un grand savant}

En effet, ces marques de reconnaissance qui ont un caractère officiel, parfois convenu, donnent à voir l'image que Joseph Déchelette avait laissée auprès de ses collègues étrangers, alors que la guerre déchirait l'Europe. Parmi ces témoignages, deux catégories peuvent être distinguées: la première concerne les lettres de condoléances qui furent adressées à sa veuve par les sociétés savantes auxquelles appartenait le conservateur roannais; la seconde est constituée principalement des articles nécrologiques qui furent publiés dans des revues scientifiques ou même dans des journaux d'audience nationale et régionale.

Commençons par les lettres de condoléances qui furent envoyées par les sociétés savantes. Dès le 23 octobre 1914, c'est la Société suisse de préhistoire ${ }^{7}$ qui fait parvenir ses condoléances signées de la main de son Président Alfred Cartier et de son secrétaire Eugène Tatarinoff:

Les travaux admirables que Joseph Déchelette avait consacrés à l'archéologie préhistorique et romaine lui avaient assuré partout une célébrité méritée; par sa vaste érudition et la sûreté de son jugement, chacun le reconnaissait comme un maittre auquel on était heureux de recourir dans les cas difficiles, et l'accueil toujours si cordial de ce collègue si loyal et si bon ajoutait encore à l'autorité du savant.

Nous avions saisi avec joie l'occasion de lui donner un faible témoignage de notre estime et de notre gratitude en lui décernant le titre de membre d'honneur de la Société suisse de Préhistoire. Nous l'avions vu à l'œuvre au congrès de Genève où il avait tenu une si large place, et nous sommes atteints d'une manière particulièrement douloureuse par la perte irréparable que vient de faire la science en la personne de notre cher et regretté collègue $^{8}$.

Le souvenir du congrès international d'anthropologie et d'archéologie préhistorique de Genève qui s'était tenu en 1912 a indéniablement assis la réputation et l'autorité scientifique du conservateur comme en témoignent d'autres lettres.

Le 6 novembre 1914, c'est l'Institut d'Estudis Catalans qui envoie une lettre signée par son Président A. Rubio y Iluch et son secrétaire Ramon d'Aloi, lettre dans laquelle est rappelée la visite de Joseph Déchelette avec son épouse deux ans plus tôt ${ }^{9}$. D'autres hommages parviennent à Roanne l'année suivante. Une lettre de la Society of Antiquaries of London ${ }^{10}$ signée par son secrétaire C.R. Reed pour le compte de son Président et du Conseil souligne:

7 J. Déchelette y avait été admis comme membre d'honneur le 18 octobre 1913.

8 Société suisse de préhistoire à Mme Déchelette, lettre du 23 octobre 1914.

9 Il y fut effectivement intégré en 1912, d'où son séjour à Barcelone.

10 Il y entre comme membre d'honneur le 2 juin 1910. 
[His] published works mark a definite epoch in the study of Roman ceramics and of Prehistoric and Celtic civilizations in Europe, to mention only a part of his achievements, and will remain indispensable to his successors and a lasting monument to himself ${ }^{11}$.

De la même manière, un hommage collectif lui est rendu par quatorze éminents confrères suédois ${ }^{12}$ le 20 janvier 1915. Enfin une lettre signée par vingt et un membres de l'Institut impérial de Moscou sera transmise à $M^{\text {me }}$ Déchelette en 1916 par A. Héron de Villefosse ${ }^{13}$.

De nombreux hommages lui sont aussi rendus dans des revues scientifiques à travers toute l'Europe. Si tous les articles nécrologiques saluent pour l'essentiel les travaux de l'archéologue, quelques-uns permettent de situer aussi bien l'œuvre que sa personnalité dans le milieu des archéologues européens. À titre d'exemple, F. Haverfield qui rédigeait un compte rendu sur la $3^{\mathrm{e}}$ partie du Manuel ("L'époque de La Tène »), ajoute:

As I write this review, the news comes that the distinguished author of this admirable work has fallen on the field of battle. [...] It is not the first, nor is it by any means the last of the losses which the war is bringing on French and on European archaeology. But it is a singularly heavy loss. [...]

We had hoped for yet another volume from M. Déchelette - a treatise on the archaeology of Roman Gaul, which should be worthy to set beside M. Jullian's Histoire de la Gaule ${ }^{14}$.

Dans les Archives suisses d'anthropologie générale, c'est le fondateur de la revue, Eugène Pittard qui, après avoir évoqué les détails de la mort de Joseph Déchelette, rappelle:

$\mathrm{Au} \mathrm{XIV} \mathrm{XV}^{\mathrm{e}}$ congrès international d'anthropologie et d'archéologie préhistorique à Genève, en 1912, Joseph Déchelette avait tenu une large place, et tous les congressistes se rappellent non seulement ses vastes connaissances mais encore sa parfaite urbanité ${ }^{15}$.

Les collègues allemands, pourtant ennemis sur le champ de bataille, n'oublièrent pas d'honorer la mémoire de celui qui fut une cheville ouvrière dans la diffusion de leurs travaux sur la Protohistoire. Ainsi Gustav Kossina écrit dans la revue Mannus $^{16}$ :

Society of Antiquaries of London à Mme Déchelette, lettre du 11 janvier 1915. Arthur John Evans était alors président de la Society of Antiquaries of London.

Parmi eux se trouvent des correspondants de Joseph Déchelette comme B. Salin, T.J. Arné, et O. Almgren.

Institut impérial d'archéologie de Moscou (au nom de l'empereur Nicolas II) à $\mathrm{M}^{\text {me }}$ Déchelette, lettre du 2 juin 1916. Plusieurs procès-verbaux émanant de ces diverses sociétés et instituts attestent également de la tristesse que la mort du savant roannais a suscitée chez beaucoup de ses pairs: voir ceux de la Society of Antiquaries of London (vol. XXVII), ou encore ceux de la Society of Antiquaries of Scotland $(50,1915)$.

F. HAVERfIELD, Journal of Roman Studies 4-2 (1914), p. 232-233.

E. PitTard, Archives suisses d'anthropologie générale, 1914-1915, p. 228.

G. Kossina, Mannus 6 (1914), p. 346-347. 
Seit langem stand er unstreitig an der Spitze der französischen Vorgeschichtsforschung, obwohl er von dem Kreise amtlicher Vertreter dieser Wissenschaft in Paris, wie sie in der Académie des inscriptions vereinigt sind, sich fern hielt.

C'est encore en Allemagne, qu'une revue, aussi importante que la Prähistorische Zeitschrift, à laquelle Joseph Déchelette avait contribué, lui consacre, dès 1914, un hommage appuyé signé par C. Schuchhardt ${ }^{17}$.

Des hommages sont aussi rendus dans des journaux d'audience nationale ou régionale. Eugène Tatarinoff revient dans les Nouvelles de Bâle $e^{18}$ sur le Manuel mais aussi sur l'homme:

Quel travail de géant! Il n'y a rien d'étonnant à ce que cet érudit modeste se soit lié avec nos savants suisses contemporains, tels que Heierli, Viollier, Vouga. Tous ceux qui ont eu besoin d'être documentés ne se sont jamais adressés en vain à Déchelette.

Ainsi, lorsqu'au cours des fouilles de la Tène, il s'élevait un doute ou une question à résoudre, on concluait à l'unanimité: "Il faut en faire part à Déchelette ", ou bien " Il faut consulter Déchelette ».

La Société Suisse pour l'étude de la préhistoire l'a compté parmi ses premiers membres et lorsqu'il s'est agi d'honorer dans toute l'Europe les savants éminents le nom de Déchelette a figuré en première ligne.

L'auteur de cet article ne peut se rappeler sans émotion la lettre sympathique reçue du Conservateur du Musée de Roanne (poste officiel de Déchelette, mais suffisant à sa modestie) et accusant réception de sa nomination.

Le grand maître de la préhistoire est mort pour sa patrie à l'âge de 52 ans, peu de temps après Heierli, le grand érudit suisse de la culture préhistorique (ils étaient tous deux en relations suivies). Il n'a pu terminer l'œuvre de sa vie, l'archéologie gallo-romaine, qui eut pu tant servir à nous, Suisses. Elle restera sans doute inachevée.

Même dans le journal Copenhague, la mort du conservateur roannais est annoncée par un de ses correspondants danois, Frederik Poulsen, directeur de la Glyptothèque Carlsberg ${ }^{19}$. Enfin M.J. Linan y Heredia laisse place à des souvenirs personnels dans la notice qu'il rédige pour le Correo español publié le 11 novembre 1914:

17 C. SchuchHhardt, Prähistorische Zeitschrift (1914), p. 368-369: « Er, der freie freundliche Mann, der zu jeder Auskunft, zu jeder Mitarbeit bereit war, der gute Kamerad bei Museums-und Ausgrabungsbesuchen, der Freund deutscher Wissenschaft wie kaum ein anderer in Frankreich!»

18 E. Tatarinoff, Nouvelles de Bâle, 24 octobre 1914.

19 "Son œuvre, le Manuel d'archéologie est un maître-livre, qui doit être connu par tous et spécialement par ceux qui s'occupent de la préhistoire du Danemark. Il y a une année, j'ai eu une correspondance avec lui concernant une série de conférences qui devaient avoir lieu à la Glyptothèque. Malheureusement, le projet ne fut pas réalisé, bien que Déchelette y fût très intéressé " (Copenhague, 5 décembre 1914). 
J'eus l'honneur et le plaisir de connaître Joseph Déchelette au cours d'une mémorable excursion au château du Marquis de Cerralbo, transformé en hôpital, qui se trouve à Santa Maria de Huerta. [...] Celui-ci, accompagné de sa femme, personne aimable et d'une grande intelligence, vint exprès de Roanne où il habitait. Il me suffit de quelques jours passés en compagnie de Déchelette pour me convaincre qu’à ses qualités de savant s'alliaient celles d'un parfait gentilhomme, dont il est précieux de posséder l'amitié.

Ces divers exemples témoignent de la réputation que s'était acquise l'œuvre dans la communauté scientifique de l'époque, ainsi que des qualités humaines du savant. Mais au-delà des compliments et des formules toutes faites ${ }^{20}$, ils sont surtout le reflet des efforts accomplis par le savant roannais dans la diffusion des découvertes et des travaux menés dans des régions qui restaient à l'écart des préoccupations d'autres grands savants français, car cette diffusion, sans son aide, n'aurait probablement pas été garantie. Dans le livre d'or qui fut publié par François Déchelette, plusieurs textes écrits à l'occasion du centenaire de sa naissance soulignent encore l'importance de l'œuvre dans les études sur la préhistoire ${ }^{21}$.

Tout aussi intéressantes sont les lettres de condoléances adressées à la veuve par certains correspondants, car ces lettres privées rendent compte de liens plus étroits, souvent de réelle amitié. Parmi les collègues suisses, citons un extrait de la lettre de David Viollier ${ }^{22}$ avec qui les relations s'étaient renforcées juste avant la guerre:

C'est avec une très profonde et très douloureuse émotion que je viens d'apprendre la triste nouvelle de la mort de M. Déchelette. Il meurt en héros; il a offert volontairement sa vie pour sa patrie, et cette belle mort ajoutera encore au souvenir qu'il nous laisse. C'était pour nous tous, qui nous occupons d'archéologie, un plaisir d'être en relation avec M. Déchelette, et il laisse le souvenir du plus aimable et du plus dévoué des confrères. Dans un petit monde où la jalousie règne en maîtresse, lui seul fut toujours parfaitement

20 Nous sommes dans un registre très différent de ce qu'a pu observer M-A Kaeser concernant les nécrologies consacrées à Edouard Desor qui furent rédigées selon un schéma narratif institué par E. Desor lui-même: M.-A. KAESER, L'univers du préhistorien. Science, foi et politique dans l'auvre et la vie d'Edouard Desor (1811-1882), Paris, 2004, p. 21. Dans le cas de Joseph Déchelette, il n'a laissé aucun manuscrit autobiographique et comme le prouvent certaines lettres, c'est sa mort inattendue qui a suscité ces réactions.

Professeur d'archéologie préhistorique à l'université d'Edimbourg, Stuart Pigott écrit en février 1960: «Dans la contribution de Déchelette à la préhistoire, un des aspects les plus significatifs est son insistance sur l'idée que la France ne peut être étudiée comme une unité isolée, mais seulement en rapport avec la préhistoire du reste de l'Europe et souvent du proche Orient également. Cette importance donnée au caractère international de la préhistoire était en avance sur son temps. Elle contribue à donner l'impression de modernité et de parenté avec les savants d'aujourd'hui, que l'on éprouve du commencement à la fin de la contribution massive que Joseph Déchelette a apportée à notre science. »(DÉCHELETTE, Livre d’or, p. 104). Pour une présentation plus détaillée des collègues et amis suisses de Joseph Déchelette, voir infra. 
bon et serviable pour tous ses collègues. Il laisse derrière lui un vide considérable, car il occupait une place que nul autre ne puisse remplir dans les sciences. Avec lui disparaît un de nos maîtres les plus écoutés. Il meurt avant d'avoir accompli toute sa tâche, mais ses travaux demeureront comme des modèles de probité scientifique, de clarté. Je conserverai des trop courtes années pendant lesquelles j'ai entretenu avec M. Déchelette de si cordiales relations le meilleur souvenir ${ }^{23}$.

Alfred Cartier semble lui aussi profondément affecté par cette disparition:

Bien chère Madame, La nouvelle que je viens de lire m'a frappé en plein cœur. Il n'y a pas hélas de consolations humaines pour une douleur comme la vôtre et je n'ai jamais mieux senti combien elles sont vaines et misérables, mais j'ai besoin de vous dire que votre deuil est le mien, que j'en éprouve toute l'étendue et l'amertume et que jusqu'à ma dernière heure, je pleurerai l'ami si cher et si fidèle auquel chaque année je me sentais plus uni par des liens d'étroite affection. Je l'aimais et je l'admirais dans sa vaste et solide érudition, sa puissance de travail, la lucidité et la pénétration de son esprit, la rigueur de sa méthode et la belle ordonnance de ses travaux. La science, non pas seulement française mais universelle fait en lui une perte irréparable et de cela aussi, je demeure accablé et inconsolable ${ }^{24}$.

Nous pourrions multiplier les témoignages de tristesse et de compassion qui furent alors adressés, mais celui d'Edoardo Galli ${ }^{25}$ prend un sens très particulier. En effet, dans sa lettre de condoléances, il rappelle à Mme Déchelette une visite que lui fit son époux sur le site de Fiésoles et la conversation qu'il eut avec son collègue et ami allemand le professeur August Oxé:

Ricorda Ella, illustre Signora, che cosa diceva ridendo il Prof. Déchelette col suo amico Prof. Oxé percorrendo la breve salita dagli scavi alla cittadina di Fiesole? Diceva che entrambi erano capitani della riserva, rispettivamente della Francia e della Germania, e che un giorno forse si sarebbero trovati avversari sul campo di battaglia! Quella, purtroppo, fu una profezia che si è avverata a breve scadenza! ${ }^{26}$

Ce souvenir qui remonte à un séjour italien probablement daté de 1913 montre à quel point l'archéologue devait redouter le jour où ses amis allemands deviendraient ses propres ennemis sans qu'il n'y puisse rien ${ }^{27}$.

23 D. Viollier à Mme Déchelette, lettre du 19 octobre 1914.

24 A. Cartier à Mme Déchelette, lettre du 14 octobre 1914.

25 Edoardo Galli, archéologue italien, était alors inspecteur du Musée royal de Florence.

26 E. Galli à Mme Déchelette, lettre du 25 février 1915.

27 Sur ces rapports avec l'Allemagne à travers l'exemple de la correspondance de Franz Cumont: C. Bonnet, Le "Grand atelier de la science ». Franz Cumont et l'Altertumswissenschaft. Héritages et émancipations, Bruxelles-Rome, 2005, p. 285-360. Pour une situation assez comparable dans le monde des écrivains et des philosophes, Marie-Claire Hoock-Demarle, L'Europe des lettres. Réseaux épistolaires et construction de l'espace européen, Paris, 2008. 


\section{Correspondants européens et échanges épistolaires: étude préliminaire}

Un premier dépouillement systématique sur l'ensemble des courriers étrangers (soit un peu moins de 800 lettres) permet de se faire une idée de la variété des échanges que Joseph Déchelette avait entretenus tout au long de sa carrière. Sur l'ensemble des 211 correspondants étrangers au sens large, la majeure partie est européenne ${ }^{28}$. Le corpus a été analysé et traité en partie à ce jour, et les données sont progressivement enregistrées dans un système de gestion de base de données relationnelle ${ }^{29}$. Certains résultats seront exploités dans cette étude, mais il ne s'agit encore que d'une approche préliminaire.

\section{Remarques générales}

Pour ce qui concerne la répartition par nationalité des correspondants, plus de 30\% sont d'origine allemande; les correspondants suisses, italiens, anglais, belges et espagnols sont proportionnellement moins nombreux ${ }^{30}$. Il faut noter qu'une bonne moitié de ces correspondants - toutes nationalités confondues - appartient à des institutions (musées, sociétés savantes et universités), ce qui augure bien de la possibilité de retrouver la correspondance active. Enfin, beaucoup sont cités dans le Manuel, ce qui conforte l'hypothèse selon laquelle la correspondance a joué un rôle important dans l'élaboration et la rédaction finale de son ouvre ${ }^{31}$.

L'activité épistolaire de Joseph Déchelette montre aussi une extension progressive du nombre de lettres à partir de 1901, qui s'explique sans doute par son investissement accru dans le domaine scientifique, en particulier dans les fouilles de Bibracte ${ }^{32}$. Néanmoins cette activité semble se ralentir dans les années 1906 et 1907, années

28 En effet, la correspondance comprend les courriers de deux Américains: J.H. Breasted (université de Chicago) et F.S. Chapin (sociologue qui enseignait au Smith College Massachusetts).

29 Cette base de données créée par Laurent Noguès devrait être bientôt mise en ligne. Elle peut être interrogée à partir des correspondants et des lettres.

30 On compte précisément 66 correspondants allemands. Pour le reste, on recense: 22 correspondants suisses, 21 Italiens, 20 Anglais, 16 Espagnols et 15 Belges.

31 Un relevé systématique a permis d'établir qu'en règle générale c'est plus de la moitié des correspondants qui sont cités dans l'œuvre. Sur l'usage des correspondances dans la construction du savoir: C. Bonnet et V. KRINGS, "Échos à la réception des Textes et Monuments figurés relatifs aux mystères de Mithra dans la correspondance de Franz Cumont", in C. Bonnet et V. KRINGs (éds), S'écrire et écrire sur l'Antiquité. L'apport des correspondances à l'histoire des travaux scientifiques, Grenoble, 2008, p. 303-309. Il publie en 1903 chez l'éditeur Picard une étude exhaustive des opérations menées sur le site: J. Déchelette, L'oppidum de Bibracte, Paris, Picard, 1903. 
durant lesquelles il doit reprendre son poste dans l'entreprise familiale ${ }^{33}$. On peut aussi constater qu'il existe un lien entre la préparation des publications et l'intensité des échanges épistolaires: c'est très net avant la publication de son ouvrage consacré aux Vases céramiques ornés de la Gaule romaine ${ }^{34}$ et aussi dans la période qui précède la rédaction des troisième et quatrième volumes du Manue ${ }^{35}$. On est également frappé par la régularité dans les échanges: à une lettre qui lui est adressée, Joseph Déchelette répond dans la semaine qui suit, pratique qui garantissait une circulation rapide des nouvelles et des informations scientifiques.

Les échanges épistolaires passent majoritairement par le français, mais certains correspondants n'hésitèrent pas à lui écrire dans leur langue voire dans leur langue maternelle et en français ${ }^{36}$. Il faut toutefois insister sur le fait que Joseph Déchelette avait une certaine prédilection dans l'apprentissage des langues étrangères, prédilection à laquelle avait contribué son activité de " commis voyageur ${ }^{37}$ " mais aussi une ouverture d'esprit et une curiosité qui lui étaient propres. En moins de dix années, il apprit l'italien, l'anglais, l'espagnol et le latin, et se mit au grec ancien et au tchèque ${ }^{38}$. Cette facilité d'apprentissage et une activité professionnelle qui l'amena à sillonner toute l'Europe jouèrent sans nul doute un rôle essentiel dans l'élaboration de son œuvre. Certains de ces correspondants furent sensibles à ce goût de Joseph Déchelette pour les langues étrangères. C'est le cas de l'archéologue portugais José Fortès qui, écrivant dans un français parfait, se plaignait:

33 En effet, alors qu'il consacrait tout son temps à l'archéologie, des malheurs familiaux le rappellent aux affaires à partir du mois d'août 1906: son frère meurt et son neveu Victor, qui doit succéder à son père à la tête de l'entreprise, est atteint d'une grave maladie. Les années qui suivent sont aussi un cortège de deuils: un de ses neveux disparaît en 1908 et sa mère en 1909.

34 J. Déchelette, Les vases céramiques ornés de la Gaule romaine, Paris, Picard, 1904.

35 J. DéChelette, Manuel d'archéologie préhistorique, celtique et gallo-romaine. Tome II: Archéologie celtique ou protohistorique. $2^{e}$ partie: Premier âge du Fer ou époque de Hallstatt, Paris, 1913 (volume 3); Manuel d'archéologie préhistorique, celtique et gallo-romaine. Tome II: Archéologie celtique ou protohistorique. $3^{e}$ partie: Deuxième âge du Fer ou époque de La Tène, Paris, 1914 (volume 4).

36 Dans une lettre de remerciement datée du 20 octobre 1910, José Leite de Vasconcellos lui écrit en portugais et recourt à quelques traductions en français pour les expressions difficiles.

37 Pour reprendre le beau titre de M-S BinÉTRuY, "Joseph Déchelette, commis voyageur de l'Europe ", dans Réseaux culturels européens. Des constructions variées au cours du temps, (sous la direction de R. Deloince et G. Pajonk), $125^{\mathrm{e}}$ congrès national des sociétés historiques et scientifiques, Lille, 2000, p. 173-188.

38 M.-S. BinÉTruy, De l'art roman à la Préhistoire. Des sociétés savantes à l'Institut, itinéraires de Joseph Déchelette, p. 60; et aussi "Joseph Déchelette, commis voyageur de l'Europe", p. 179. 
Malheureusement nos ouvrages ne sont pas assez connus au dehors, peut-être parce que nous ne les écrivons que dans notre langue, si peu connue ${ }^{39}$.

Le collègue portugais évoque dans un autre courrier les difficultés que ses collègues et lui ont rencontrées avec leurs homologues allemands qui ne faisaient, semble-t-il, aucun effort pour se faire comprendre ${ }^{40}$. De fait, cette aptitude fut mise au service de la démarche originale empruntée par Joseph Déchelette pour écrire une synthèse sur la Protohistoire à l'échelle européenne. Cette dimension pourrait s'apparenter à celle d'un encyclopédiste soucieux d'embrasser l'ensemble des données archéologiques de son temps, quelles que fussent leur provenance géographique et les difficultés linguistiques, mais elle apparaît aussi comme le plus évident témoin d'une appréhension de l'étude de l'âge du Fer qui était finalement à contre-courant d'approches plus nationalistes ${ }^{41}$.

Les formules de salutation qu'utilisaient les correspondants pour s'adresser à leur destinataire sont aussi un bon indice de la nature des rapports épistolaires que le conservateur de Roanne avait noués. Ces rapports sont avant tout professionnels mais des relations plus étroites se sont tissées au fil des échanges : c'est le cas de la correspondance avec le marquis de Cerralbo dont la formule de salutation passe du "Monsieur» à «Cher Monsieur et ami » en moins d'un an. Cette proximité épistolaire apparaît aussi dans les échanges avec Alfred Cartier, le directeur du musée de Genève, mais seulement au bout de quatre années de correspondance suivie. Les correspondants italiens sont eux plus enclins à donner des titres emphatiques, notamment celui d' "Illustre Professore ", alors que Joseph Déchelette n'appartenait pas au milieu universitaire. D'autres conserveront une certaine distance comme Oscar Montelius qui n'utilise que la formule de "Cher Monsieur ", bien que les lettres soient toujours très courtoises et amicales. Lorsque les liens épistolaires se resserrent, ils témoignent certes d'une collégialité entre le conservateur de Roanne et ses homologues européens, mais ils prouvent parfois que ces liens ont laissé place à des amitiés durables.

\section{Analyse préliminaire de quelques « réseaux »}

En l'état de notre dépouillement, nous limiterons cette analyse à quelques " réseaux » de correspondants, plus particulièrement aux correspondants anglo-saxons, suisses, espagnols et portugais. Ces exemples peuvent aider à comprendre comment s'est structuré autour de Joseph Déchelette un réseau relationnel scientifique à l'échelle européenne.

39 J. Fortes à Joseph Déchelette, lettre du 08/02/1905.

"Une mimique très compliquée, et des petits lambeaux du vieux latin et de toutes les modernes langues européennes... voilà notre extraordinaire moyen de communication. C'était affreux et un peu comique... » (J. Fortes à Joseph Déchelette, lettre du 25 février 1905).

41 Il s'agit là d'un des aspects fondamentaux de son œuvre que nous développerons dans une prochaine étude. 
Parmi les 28 correspondants anglo-saxons repérés trois d'entre eux ont été des interlocuteurs privilégiés de Joseph Déchelette ${ }^{42}$. Le premier d'entre eux par la quantité de lettres conservées est Horace Sandars ${ }^{43}$, archéologue amateur, responsable d'une exploitation de mines en Espagne. Il entretint une correspondance suivie avec l'archéologue roannais entre 1902 et 1914, correspondance qui s'explique peut-être par une affinité professionnelle, l'un et l'autre appartenant au monde de l'industrie. Horace Sandars, qui devint un spécialiste de la péninsule ibérique, entra en contact avec Joseph Déchelette sur les conseils du directeur du musée de Reading qui lui avait signalé la découverte, dans les environs de Roanne, de moules de fabrication. H. Sandars se proposait de se rendre à Roanne mais il lui faudra attendre le mois de juin 1912 pour que cette visite soit effective. Les échanges avec le conservateur des antiquités britanniques et d'ethnologie du British Museum, Reginald Allender Smith ${ }^{44}$ commencèrent en 1904 et se poursuivirent jusqu'en 1914 avec une certaine régularité. Après une première demande d'informations bibliographiques, R.A. Smith avait à nouveau contacté son collègue roannais pour le remercier de la part de son supérieur hiérarchique C.H. Read à propos de divers renseignements, puis pour le solliciter sur plusieurs questions, car il était chargé d'établir un catalogue des antiquités de l'âge du Fer pour le musée. Quant à l'archéologue irlandais George Coffey ${ }^{45}$, il fut un autre partenaire de choix comme en témoignent les recensions faites par Joseph Déchelette dans la revue L'Anthropologie ${ }^{46}$.

Les échanges épistolaires entre Joseph Déchelette et ses collègues des musées helvétiques ont été particulièrement intenses et riches sur le plan scientifique. Sur un total de vingt-deux correspondants, il faut encore distinguer trois personnalités: Alfred Cartier, Albert Naef et David Viollier. Curieusement les relations avec Paul Vouga ${ }^{47}$

42 Il est à noter que le fonds recèle une lettre d'Arthur John Evans en date du 5 janvier 1910; elle fait suite à une demande transmise par R. A. Smith à propos de lames de bronze.

43 1852-1922. Nous avons recensé 24 lettres pour douze ans d'échanges.

44 1873-1940. Il a été conservateur des Antiquités britanniques et médiévales et d'Ethnographie de 1898 à 1938. La correspondance compte 15 lettres, mais il semble que des lettres manquent par recoupement avec d'autres correspondances, notamment la lettre à A.J. Evans précédemment citée.

45 George Coffey 1857-1916. Nous avons recensé 11 lettres.

46 J. DÉchelette, "Compte rendu de Coffey George: Intercourse of Gaul with Ireland before the first Century (1910)", L'Anthropologie, 22, 3, 1911, p. 336-337; "Une nouvelle interprétation des gravures de New Grange et de Gavr'Iris”, L'Anthropologie, 23, 1, 1912, p. 30-52.

47 1880-1940. Comme son père Émile, Paul Vouga eut la responsabilité des fouilles du site de La Tène et devint l'une des figures majeures de l'archéologie en Suisse. Voir M.-A. KaESER, "De la mémoire à l'histoire: Paul Vouga et l'archéologie préhistorique au début du Xxe siècle”, dans M.-A. KAESER (dir.), De la mémoire à l'histoire: l'œuvre de Paul Vouga (1880-1940). Des fouilles de La Tène au "néolithique lacustre ", Neuchâtel, 2006, p. $11-31$. 
furent plus réduites bien que l'intérêt de l'archéologue roannais pour le site de la Tène fût constant ${ }^{48}$. La première lettre avec Albert Naef ${ }^{49}$ date du 14 janvier 1901, mais les deux hommes se connaissaient déjà puisqu'ils s'étaient rencontrés au congrès de Mâcon ${ }^{50}$, en 1899. Leurs échanges furent assez réguliers jusqu'en 1912, année après laquelle nous ne trouvons plus trace de courrier entre les deux hommes. Quant à Alfred Cartier ${ }^{51}$ et David Viollier ${ }^{52}$, leurs correspondances avec l'archéologue roannais débutent respectivement en 1908 et 1909, et elles se maintiendront de façon très régulière jusqu'à la veille du conflit mondial. Ces liens se resserrèrent du fait des visites fréquentes de Joseph Déchelette à ses collègues suisses (au moins une fois par an entre 1909 et 1913) et du rôle important qu'il joua lors du congrès de Genève en 1912.

Concernant les correspondants espagnols et portugais, nous retiendrons ici les échanges entretenus avec Manuel Cazurro, le marquis de Cerralbo, José Fortès et José Leite de Vasconcelos. C'est en 1910 que semblent commencer les relations épistolaires avec Manuel Cazurro ${ }^{53}$ et le marquis de Cerralbo ${ }^{54}$, l'un et l'autre étant impliqués dans des campagnes de fouilles majeures à cette époque: celles d'Ampurias pour le premier et les sites du Haut Jalón pour le second. Ces échanges seront réguliers jusqu'en 1914 avec M. Cazurro et jusqu'en 1913 avec le marquis de Cerralbo. Quant à José Fortes et José Leite de Vasconcellos ${ }^{55}$, les premiers contacts se nouent en 1903, de façon assez originale. En effet, c'est de sa propre initiative que l'avocat et archéologue José Fortes se présente à Joseph Déchelette en lui indiquant qu'il étudie la palethnologie de son " petit pays, le Portugal, cette région si intéressante dans ses multiples stations pré- et

48 En effet, il existe seulement trois lettres de Paul Vouga, dont deux pour l'année 1911 et une datée de 1913 qui concerne la visite de J. Déchelette à Neuchâtel. 1862-1936. Il fut à partir de 1899 chef du Service des Monuments historiques du Canton de Vaud puis professeur d'archéologie à l'université de Neuchâtel en 1905. Nous avons dénombré 27 lettres. Plus précisément ce fut au $66^{\mathrm{e}}$ congrès archéologique de Mâcon, le 18 juin 1899, où J. Déchelette fit la lecture de l'étude de J.L. Pič sur l'oppidum de Stradonice. 1854-1921. Alfred Cartier fut directeur du musée de Genève et président de la Société suisse de préhistoire (1901). Le fonds contient 22 lettres.

1876-1965. Il fut d'abord l'assistant d'Albert Naef avant de devenir le spécialiste du second âge du fer suisse. Nous avons compté 16 lettres dans le fonds. C'est par l'intermédiaire d'Alfred Cartier qui lui demande d'accueillir J. Déchelette lors d'une de ses visites que tous deux vont faire connaissance (lettre du 15/07/1909).

1865-1935. Sur M. Cazurro Ruiz, voir récemment I. Vallvé Albiol, "Manuel Cazurro Ruiz, pioner d'Empúries i colleccionista", Annals de l'Institut d'Estudis Gironins, 46 (2005), p. 367-405. Le fonds contient 12 lettres.

54 Enrique de Aguilera y Gamboa, Marqués de Cerralbo (1845-1922), grande figure de l'archéologie en Espagne. Dans le fonds nous avons trouvé 22 lettres dont une adressée à Mme Déchelette.

55 1858-1941. Il fut le fondateur et le directeur du Musée Ethnographique Portugais. On recense 13 lettres de sa main dans le fonds de Roanne. 
protohistoriques $^{56}$ ». Quant à José Leite de Vasconcellos, c'est Camille Jullian qui fait suivre directement le courrier qu'il a reçu en précisant à Joseph Déchelette qu'il lui transmet un mot du " bibliothécaire de Lisbonne, charmant homme, fort instruit, et dont vous devez du reste connaître les travaux sur les antiquités préhistoriques et autres au Portugal ${ }^{57}$ ".

À travers les lettres de ces différents correspondants, on peut discerner plusieurs types d'informations: les informations purement scientifiques en relation directe ou non avec les publications de Joseph Déchelette; les « querelles » et critiques adressées à l'encontre de certains collègues; les recommandations sollicitées auprès du conservateur de Roanne; enfin les affaires plus personnelles qui témoignent encore de relations qui dépassent les pratiques d'une sociabilité uniquement scientifique. Quelques exemples illustreront ces diverses catégories et permettront de comprendre aussi comment et pourquoi la correspondance peut devenir un champ d'exploration incomparable.

Une grande part du contenu des lettres relève du domaine de l'information scientifique stricto sensu, qu'il s'agisse de découvertes, de campagnes de fouilles ou encore de renseignements sur des objets précis. Cette information passe souvent par l'envoi de photographies ou de longs rapports illustrés de croquis qui permettent au destinataire de se rendre compte de la valeur de certaines trouvailles. Ainsi le marquis de Cerralbo n'hésite pas à donner régulièrement un rapport de ses fouilles qu'il soumet à l'appréciation du spécialiste de l'âge du Fer et dont la synthèse sera publiée ${ }^{58}$. De la même manière, Joseph Déchelette recourt au service de David Viollier pour la correction d'épreuves d'une publication qu'il avait rédigée en allemand ${ }^{59}$. Il est à noter que beaucoup de correspondants étaient aussi de bons dessinateurs, le dessin tout comme la photographie, jouant un rôle probatoire majeur ${ }^{60}$. L'échange de publications - tirés à part, ouvrages gracieusement donnés - et le prêt de livres dont certains appartiennent à la remarquable bibliothèque qu'il s'est constituée renforcent enfin la diffusion scientifique des découvertes d'un pays à l'autre et peuvent contribuer à l'avancement de certains travaux.

L'espace épistolaire est aussi marqué du sceau de la réserve. Les critiques à l'égard de collègues sont assez fréquentes et parfois acerbes. Dans un passage où il évoque la parution d'un livre sur les fouilles menées sur le site de Münsingen-Rain, Albert Naef fait quelques commentaires sur son auteur, Jacob Wiedmer-Stern, jugeant qu'il a

56

José Fortes à Joseph Déchelette, lettre du 08 février 1903. Il existe 28 lettres et cartes de sa main.
Camille Jullian à Joseph Déchelette, lettre non datée (avant ou tout début de 1903).

J. Déchelette, "Les fouilles du marquis de Cerralbo", Comptes Rendus de l'Académie des Inscriptions et Belles Lettres, 1912, p. 433-443. Joseph Déchelette avait été élu membre correspondant de l'Institut le 22 décembre 1912.

David Viollier à Joseph Déchelette, lettre du 10 juin 1912.

S. Lewuillon, "Archaeological illustrations: a new development in 19th century science", Antiquity, 76, 2002, p. 223-234. 
fouillé « trop vite » et " [qu'] on sentait le conservateur de musée, impatient de récolter le plus et le plus vite ». Il prend soin toutefois de souligner: "Cela entre nous ${ }^{61}$ ". L'archéologue portugais José Fortes fait lui aussi part de ses critiques au sujet du livre de Pierre Paris, Essai sur l'art et l'industrie de l'Espagne primitive paru en 1903 et qui fut recensé par Salomon Reinach ${ }^{62}$ : selon lui, P. Paris ne connaît pas les travaux de Martins Sarmiento qui de longue date avait posé et confirmé l'hypothèse des contacts entre l'Espagne et la civilisation mycénienne ${ }^{63}$ ! Quelques collègues français et proches de J. Déchelette semblent cristalliser les critiques de certains correspondants, à l'exemple de Salomon Reinach, jugé « trop fonceur " par Albert Naef ${ }^{64}$, ou critiqué pour son attachement au pantotémisme ${ }^{65}$. Certaines querelles ne semblent pas non plus éteintes, comme en atteste une diatribe d'Alfred Cartier contre Camille Jullian à propos de l'âge du Bronze ${ }^{66}$. Quant à Joseph Déchelette, il n'échappait pas non plus à ces disputes scientifiques mais ce sont ses propres travaux qui soulèvent parfois quelques remontrances qui restent toutefois courtoises ${ }^{67}$.

Un autre rôle assumé par Joseph Déchelette est celui d'intermédiaire entre savants et érudits, mais aussi entre savants et personnalités politiques. Dans le premier registre, on peut citer deux exemples emblématiques de cette position bien particulière de l'archéologue. En effet, c'est par une lettre qu'Horace Sandars lui demande de le recommander auprès de Louis Capitan et de Charles Buttin, son but étant d'avoir accès à la collection de bronzes ibériques de Georges Pauilhac ${ }^{68}$. L'accès lui en sera facilité par le

61 Albert Naef à Joseph Déchelette, lettre du 12 février 1911. À l'inverse il juge «Vouga, à Neuchâtel " comme un "très gentil garçon, qui mérite d'être encouragé et poussé. "

Le compte rendu de S. Reinach se trouve dans la Revue archéologique 5-1 (1905), $4^{\mathrm{e}}$ série, p. $156-160$. Albert Naef à Joseph Déchelette, lettre du 12 février 1911.

65 Alfred Cartier à Joseph Déchelette, lettre du 4 mars 1909.

Alfred Cartier à Joseph Déchelette, lettre du $1^{\text {er }}$ janvier 1913: «Vous avez lu sans doute, dans le dernier $n^{\circ}$ de la Revue des Études anciennes, la note de Jullian où il rapproche, temps et caractères, les alignements de Carnac de la nécropole d'Aguilar et où, malgré toutes vos démonstrations, il fourre des Ligures en Armorique. C'est proprement désolant. Ainsi l'effort énorme, les découvertes décisives accomplis depuis Bertrand, l'existence de cette longue période du Bronze, tout cela n'existe pas ou se nie implicitement. Nul plus que moi n'admire le talent et le mérite de notre ami, mais il y a décidément chez lui incapacité radicale à admettre autre chose que les dires des historiens anciens qui auront toujours raison contre les faits archéologiques, et c'est d'autre part, toujours par la surface et non dans leur essence qu'il saisit les rapports des choses. "

67 Aimé Rutot à Joseph Déchelette, carte du 10 octobre 1908. Sur une carte de visite personnelle, le préhistorien belge accuse réception de l'envoi du premier tome du Manuel mais il précise en se mettant à distance: "Il regrette de n'être d'accord avec l'auteur sur aucun point relativement à la question des Eolithes [...] et renouvelle son offre de prouver à l'évidence, pièces en mains, tout ce qu'il annonce. " 
truchement de J. Déchelette et $\mathrm{H}$. Sandars le remerciera avec force compliments dans une de ses lettres ${ }^{69}$. Grâce au marquis de Cerralbo qui avait présenté sa candidature, Joseph Déchelette avait été accepté comme membre correspondant de l'Académie royale de Madrid dès $1910^{70}$. De la même manière le conservateur de Roanne permet au marquis d'entrer en contact avec Eugène Pittard qui s'occupait de l'organisation du colloque de Genève, ainsi qu'avec l'Académie des Inscriptions et Belles Lettres ${ }^{71}$. Dans le second registre, plus politique, Albert Naef recourt à ses services pour être recommandé auprès de M. de Saint-Arroman au ministère de l'Instruction publique ${ }^{72}$.

Certaines lettres contiennent des notes plus personnelles: les deuils, la maladie de proches, mais aussi certains événements politiques sont partagés entre interlocuteurs ${ }^{73}$. Enfin certaines requêtes plus singulières sont adressées à Joseph Déchelette: ainsi José Fortes lui demande un portrait pour connaitre "l'homme physique ${ }^{74}$ ». Un an plus tard, le conservateur roannais satisfera sa demande à la grande joie de l'érudit portugais ${ }^{75}$.

Enfin, il fallut renforcer cette proximité d'étude par des voyages et de fréquents séjours à Paris, où le Musée de Saint-Germain-en-Laye et son conservateur Salomon Reinach servaient de relais essentiel à nombre de correspondants. L'hospitalité et l'autorité grandissante du conservateur roannais firent bientôt du musée de Roanne un autre passage obligé pour tout savant européen et la liste est longue de tous ceux qui rendirent visite à leur collègue français ${ }^{76}$.

La thèse de M.-S. Binétruy s'intéressait à l'homme et à son milieu social et culturel. Au terme de cette première étude, qui sera complétée par d'autres dossiers documentaires ainsi que par une mise en ligne progressive des données que le fonds de Roanne recèle, plusieurs perspectives d'étude se dégagent. La correspondance a indéniablement joué un rôle déterminant dans la diffusion des découvertes scientifiques et dans la circulation des informations. La régularité des échanges a permis l'abolition des distances entre interlocuteurs et, si la dimension européenne de l'œuvre de Joseph Déchelette va de pair avec celle de sa correspondance, il serait aussi intéressant de voir comment chaque phase de

69 Horace Sandars à Joseph Déchelette, lettre du 30 juin 1912.

70 Marquis de Cerralbo à Joseph Déchelette, lettre du 11 août 1910.

71 Marquis de Cerralbo à Joseph Déchelette, lettre du 14 mai 1912: "Je vous remercie d'avoir écrit à Pittard. " Dans une lettre datée du $1^{\text {er }}$ septembre 1912, il autorise Joseph Déchelette à faire une communication consacrée à ses fouilles auprès de l'Académie; il sera lui-même invité à présenter ses résultats quelques semaines plus tard (lettre du 19 octobre 1912).

72 Albert Naef à Joseph Déchelette, lettre du 29 mai 1904.

73 Ainsi Juan Rubio de la Serna évoque, dans une lettre en date du 10 août 1908, les événements " catastrophiques » qui ont alors eu lieu à Barcelone (il s'agit des attentats anarchistes).

74 José Fortes à Joseph Déchelette, lettre du 28 février 1905.

75 José Fortes à Joseph Déchelette, lettre du 11 janvier 1906: «À titre d'échange, je vous envoie ma photographie par cette poste...".

76 Parmi eux, citons José Fortes, Horace Sandars, Alfred Cartier, ou Hans Dragendorff... 
cette correspondance s'est inscrite dans les diverses étapes de la maturation et de la rédaction du Manuel. Par le biais de cette correspondance, c'est aussi l'archéologue au cœur d'une communauté savante qu'il nous est possible d'étudier: sa place dans les réseaux européens et nationaux qui s'intéressaient à la Protohistoire et son action décisive dans le dialogue entre archéologues des quatre coins de l'Europe.

Sandra PÉRÉ-NoguÈs

Maison de la Recherche

Laboratoire TRACES (UMR 5608)

5 allées Antonio Machado

F-31058 Toulouse

perenog@univ-tlse2.fr 